of the species are printed on a large chart adjacent to the stations at which they were collected. Prof. Hickson directs attention to the rich harvest of sea-pens gathered around Amboyna, the Banda and Kei Islands, and off the south coast of Timor and Flores, and concludes that the Malayan region is the headquarters of the genera Pteroeides and Virgularia. He remarks that, although there is not sufficient information in regard to other genera to justify a similar conclusion, the facts as they stand are in accordance with the view that the Malay Archipelago is, or has been, a distributing centre of the Pennatulacea of the world. Of special interest from the point of view of geographical distribution is the occurrence of the following, all deep-sea forms: Chunella grácillima, previously known from the east coast of Africa; the genus Gyrophyllum, hitherto recorded only from the North Atlantic; and five species of Umbellula.

Anatomical and histological investigations have been made on a number of interesting points, $e . g$. (i) the ciliated radial canals, found throughout the rachis of Virgularia, which Prof. Hickson suggests are concerned with the flow of water into and disterrsion of the colony; (ii) the large mesozooids of Pennatula murrayi, the structure of which indicates that they bring about rapid expulsion of water from the principal canals; (iii) the brown ciliated tubes of this species; and (iv) the gonads-all the species examined proved to be diœecious and oviparous.

Useful keys are given to the families, genera, and species, and the memoir is illustrated by ten plates and forty-five text-figures.

Prof. Hickson is to be warmly congratulated on the completion of this important memoir, which is charac terised throughout by great care and sound judgment.

\section{EXPERIMENTAL HYDRAULICS.1}

THE small amount of evidence, which many engineers are willing to accept as satisfactory proof of some principle or empiricism used in connection with their designing, is sometimes surprising to those who combine, with engineering experience, knowledge of the more refined and rigid methods of scientific inquiry. Perhaps there is no more striking evidence of this than in connection with the formulæ used by engineers, in perfect faith, to determine the flow of water over weirs and through orifices and nozzles.

Very frequently in experimental work there is a want of precision in the results, owing to lack of appreciation of what might be called the persistence of hydraulic disturbance. In our technical colleges apparatus which is supposed to compare the loss of head in certain lengths of pipes of different form, and certainly measures something, but not that which the designer intended, is not infrequently used by students.

It is to be regretted that so little attention has been paid in this country to precise experimental hydraulics; but because of that we are so much the more indebted to those workers who, in France and the United States, have added to our experimental knowledge of this important subject.

The modern universities of the United States are issuing from their experimental stations many interesting Bulletins describing the results of special researches, and Bulletin 96 of the University of Illinois, though not by any means ambitious, is yet of sufficient importance to receive a passing notice in the columns of Nature. It describes experiments on the effect of fixing mouthpieces of different shapes on a dis-

1 "The Effert of Mouthnieces on the Flow of Water through a Submerged Short Pipe." By F. B. Seely. Rulletin No. 96. (University of Illinois.)

NO. 25 I6, VOL. IOO] charge through a short drowned pipe. The apparatus is described, and the coefficients of discharge for a sixinch short pipe without mouthpieces at either end, and with the inlet projecting and not projecting inwards respectively, as well as for different combinations of mouthpieces at inlet and outlet, are given. A bibliography of the subject is attached to the paper.

\section{ASTRONOMICAL CONSEOUENCES OF THE ELECTRICAL THEORY OF MATTER. ${ }^{1}$}

CERTAIN complications have recently been introduced into theoretical physics or physical philosophy which, though not of immediate application to engineering, should have an interest for all educated people.

The doctrine of relativity is based essentially on two negative experiments. One of these was conducted by me at Liverpool, and is fully recorded in the Philosophical Transactions of the Royal Society for ${ }_{1} 893$ and I894. The outcome of the experiment is to show that the velocity of light is not affected in the neighbourhood of rapidly moving matter; thus, in language appropriate to æther, implying that the æther is stationary in space and cannot be carried along by moving matter; that there is no viscous or frictional drag between matter and ether. The other and more famous experiment is that of Michelson and Morley, which proves that the time of a light-journey to and fro between points fixed to the earth is not affected by azimuth; which therefore appears to imply that the earth is not moving freely through the æther, as the first experiment requires, but that the adjacent æether is stagnant with respect to the earth's surface, as if a layer of some thickness were fully carried along with the earth in its motion through space.

(I must here say that this is a conclusion which, if admitted, would involve many difficulties, and would. complicate the relation between æether and matter amazingly.)

The two experiments are thus contradictory, suggesting that the wording of the conclusion in terms of æther may be wrong; and inasmuch as all experiments on the æther have so far given negative results except when there was some movement of matter relative to matter, a doctrine of relativity has arisen which begins by postulating that such experiments always will give negative results, that the properties of an æther can never be ascertained, that things go on as if space were empty, that movement of matter has no meaning except with reference to other matter, and hence that in all probability the æether does not exist. I ought perhaps to make it clear that I myself do not hold this doctrine; but on that subject I have expressed my own position in my British Association address, published by Messrs. Dent and Sons under the title "Continuity."

How the velocity of light, which is an undeniable and metrical fact, can thus be understood or systematised, without a medium possessed of definite physical properties, seems to conservative physicists a substantial difficulty at the outset. Nevertheless, they are willing to admit that questions directly addressed to the æther have always received negative replies: always except once-the measurement of the finite and definite velocity of light, both in free space and in transparent matter. Beyond this, the three salient optical phenomena-viz. the Bradley aberration, the Fizeau convection, and the Doppler change of frequency-all involve motion of matter relative to matter.

1 Abridgment of a lecture delivered to the student-members of the Institu. tion of Electrical Engineers on November 23, $19^{17}$, by Sir Oliver Lodge, F.R.S 